\title{
Safety recommendations for dentists and maxillofacial surgeons during COVID-19 pandemic
}

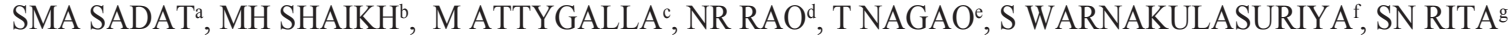

\begin{abstract}
:
Oral health professionals are at high risk of developing COVID-19 infection due to their practice of close proximity to the patients. Patients in daily dental practice can be seen with basic protection protocol. Routine and elective dental procedures should be cancelled during this pandemic situation and could be rescheduled when the situation eases. In practice, as of universal prevention strategy, all patients should be assumed as COVID-19 infected and treated accordingly. Adequate standards of hygiene and protection should be adopted for all patients to avoid potential transmission risks. During the COVID-19 pandemic, it would be crucial to categorize oral and maxillofacial surgery cases into: emergency
\end{abstract}

Introduction:

The first case of a COVID-19 was reported in Wuhan, Hubei Province, China on $31^{\text {st }}$ December 2019. ${ }^{1}$ The patient admitted to the hospital with pneumonia of unknown origin, and later tested positive for the $\beta$-coronavirus. The novel $\beta$-coronavirus was isolated and declared as the causative pathogen

a. Dr. S. M. Anwar Sadat, Associate Professor, Dept of Oral \& Maxillofacial Surgery, Dhaka Dental College, Dhaka, Bangladesh, Consultant, Square Hospitals Ltd, Dhaka

b. Dr. Mushfiq H. Shaikh, Postdoctoral Fellow, Department of Otolaryngology - Head and Neck Surgery, Schulich School of Medicine and Dentistry, Western University, London, Ontario, Canada.

c. Dr. Manjula Attygalla, Prof in Oral and Maxillofacial Surgery, Faculty of Dental Sciences, University of Peradeniya, Sri Lanka

d. Dr. Naman R. Rao, Henry M. Goldman School of Dental Medicine, Boston University, Boston, Massachusetts, USA

e. Dr. Toru Nagao, Professor and Chairman, Department of Maxillofacial Surgery, Aichi Gakuin University School of Dentistry, 2-11, Suemori-dori, Chikusa-ku, Nagoya, 464-8651, Japan

f. Dr. Saman Warnakulasuriya, Faculty of Dentistry, Oral \& Craniofacial Sciences, King's College London, WHO Collaborating Centre for Oral Cancer, London, UK

g. Dr. Sufia Nasrin Rita, Professor and Head, Dept of Orthodontics, Sapporo Dental College, Dhaka, Bangladesh. Consultant, Square Hospitals Ltd, Dhaka

Address of Correspondence: Dr. S. M. Anwar Sadat, Associate Professor, Dept of Oral \& Maxillofacial Surgery, Dhaka Dental College, Dhaka, Bangladesh. Consultant, Square Hospitals Ltd, Dhaka. Cell: +8801711156023. E-mail: an_sadat@yahoo.com case, urgent case, intermediate case and low priority cases. While there has been a growing literature suggesting the aspect of critical care associated in treating these patients, ample of evidence indicates how this COVID-19 pandemic will affect surgical practice. Here we highlight the current unfavorable situation due to COVID -19 and offer recommendations for changes to dental and maxillofacial surgical practices.

Key Words: Bangladesh, COVID-19, Dentist, Maxillofacial Surgeon, Recommendation

(J Bangladesh Coll Phys Surg 2020; 38: 197-204)

DOI: https://doi.org/10.3329/jbcps.v38i4.48981

on $7^{\text {th }}$ January, $2020^{2}$. The virus was named officially as "Severe Acute Respiratory Syndrome Coronavirus 2" (SARSCoV-2) (Coronaviridae, 2020). The World Health Organization (WHO) declared the 2019-20 coronavirus outbreak as a Public Health Emergency of International Concern (PHEIC) on $30^{\text {th }}$ January $2020^{3}$, and later as a pandemic on $11^{\text {th }}$ March $2020^{4}$. As of July $29^{\text {th }}$ a total of $169,36,074$ detected cases and 664402 deaths have been reported worldwide. In Bangladesh the total number of affected person is 321615 with total death is 4412 (as of $3^{\text {rd }}$ September 2020) (webometre).

There is no specific treatment or vaccine yet for COVID- 19. Health care providers, especially the oral health professionals, are at high risk of developing COVID-19 infection due to their practice of close proximity to the patients. The attendants can also be a source of carrying the virus without having any symptoms. In Bangladesh, as of August 10, 7442 health workers including 2542 physicians, 1860 nurses and 3040 other health workers have been infected with the corona virus, reported by Bangladesh Medical Association (BMA) in a statement. They also confirmed death of 77 physicians within this Covid situation as of $10^{\text {th }}$ August. While the transmission of COVID-19 via human exhaled droplets and direct contact is clear, the potential for aerosol transmission is a significant risk particularly for dental practices ${ }^{5}$.

COVID-19 spreads very rapidly. Though still not clearly concluded, at present, the respiratory droplet transmission mode and the contact transmission 
mode have been confirmed ${ }^{2,6}$. Whether the virus spreads through the fecal-oral route, aerosol or other methods is uncertain, and this poses greater challenges to prevent any transmission. COVID-19's potential infectiousness during the incubation period further complicates its prevention and control. According to the latest reports, the longest incubation period so far is 24 days $^{7}$, though usually considered up to 14 days.Dental practices with its specialties are at higher risk of disease transmission among oral health care providers and patients due to close contact with saliva, blood, andwater droplets ${ }^{8,9}$.

The purpose of this recommendation paper is to increase awareness among the dental health care providers about the Covid situation and support them by way of protecting themselves from being affected by Covid 19 during their clinical practice. Here we will also highlight what the specialty is facing and how it is handling the situation, in the hope that this information will be useful as a reference in the future, not only for this specialty, but also for others, should COVID- 19 or a similar global threat arise again.

\section{General practice recommendation:}

Patients attending routine dental practice can be seen with a basic protection protocol. Routine, elective dental procedures can be cancelled in the pandemic situation and can be rescheduled for a later time when the situation will be under control. Services can be limited to patients requiring urgent consultation with or without any clinical procedure and follow-up. Non-emergency visits can be replaced by audio videoconference which can easily be done as most of the people of Bangladesh are connected with mobile phones and with internet anyway. Before coming to the dental office, the patients can be screened for Covid 19.

Working hours and clinic staffs should be limited according to patient turnover and senior practitioners especially with comorbidities like Diabetes Mellitus, Hypertension, Ischemic Heart Disease, Bronchial Asthma, COPD, Chronic Kidney Disease, and Chronic Liver Disease, having immune suppressive drugs or anticancer drugs should be prevented from patient contact and service should depend on young professionals. Proper education, guidance with training and monitoring of protective measures through wearing standard masks, goggles, face shields, gloves and personal health and hand hygiene with waste disposal by all health care providers must be ensured in service areas.

In practice with universal prevention strategy, all patients should be assumed to be infected and treated accordingly. In previously infected cases, it is necessary to be ensured of 2negative COVID-19 tests separated by at least 24 hours due to the possibility of false negative results.

Adequate standards of hygiene and protection should be adopted for all patients to avoid potential transmission risks. Using mouthwash with $0.5 \%$ hydrogen peroxide, $1 \%$ povidone iodine with virus-inhibiting effect before oral procedure ${ }^{10,11}$, using rubber dam for effective isolation with strong suction to reduce pollutant spread can significantly reduce transmission of the virus in practice environment. Dental patients usually have a strong urge to spit after a procedure. COVID-19 has been isolated in infected saliva ${ }^{12}$; patient spitting could enhance the spread of the COVID-19 within the dental office.

Oral diseases have a significant interplay with systemic diseases especially among the elderly ${ }^{13}$. When managing the elderly, who may be in self-isolation during the COVID outbreak a telephone consultation with the patient's general practitioner or specialist would be desirable before prescribing over the phone.

\section{Recommendations for dental health care providers in work places:}

The members of the team should follow regular meetings about the status of Covid in the country and should be trained to participate in the process of protective measures to prevent cross contamination. Staff members can be directed to follow the Triaging to enter the department.

Their body temperature needs to be recorded at the entrance using a contactless thermometer. Restriction of entry can be recommended with staff having history of contact with a Covid positive person in the family and surroundings or having any of the symptoms like sore throat, cough, running nose, fever and breathlessness.

The health care providers should not use or carry unnecessary personal belongings e.g. Jewelery, watch or other accessories and also refrain from carrying, using and sharing mobile phones, pens, computers and common telephones, desks, or work tools, equipment, books, foods, goods that can interfere with infection control. Direct face to face verbal communication between colleagues and unnecessary crowding of excess staffs in the operating room can be restricted for them. After having foot bath $(0.1 \%$ Sodium hypochlorite solution) at the entrance, keeping distance of one meter between staffs at working times with regular interval hand wash with soap water and alcohol based 
hand rub (minimum 70\%Alcohol) are highly recommended. Besides, personal hygiene maintenance with wearing appropriate type and technique of mask, caps, goggles and face shields all the time with following respiratory etiquette and discard the used materials in the allocated closed bin need to be monitored by the team leader of the working environment.

Maintain regular housekeeping practices, including routine wiping/cleaning and disinfecting of surfaces, equipment, and other items in the work environment. The door knobs/handles, switches on lift doors shall be cleaned and disinfected every three hours. It should be a regular practice to disinfect metal surfaces including door locks, handles with minimum of $70 \%$ alcohol solution and non-metal surfaces with $0.1 \%$ sodium hypochlorite solution in a three hours interval. Office equipment, furniture and floor can be cleaned twice daily. Change of personal clothing and full shower should be followed before entering and after returning home.

Recommendation for the treatment delivery area: (I) It is recommended to treat all patients in a single room by controlling the admission of number of patients reporting to the reception.

(II) Consultation, procedure and waiting areas should be rigidly isolated. Facility of good ventilation and also mechanical ventilation should be adopted if necessary. Before starting consultation or procedure, the room should be disinfected by ultraviolet irradiation and or preferablywith a disinfectant containing 2,000 $\mathrm{mg} / \mathrm{L}$ of effective chlorine spray.

(III) Disinfect the object surfaces and the floor with disinfectant $2,000 \mathrm{mg} / \mathrm{L}$ of effective chlorine twice daily, and disinfect as soon as possible after contamination. Pay special attention to high-frequency contact surfaces e.g.instrument panels, handles, buttons etc.

(IV) After treating each patient, chair should be wiped with $75 \%$ ethanol or $2,000 \mathrm{mg} / \mathrm{L}$ disinfectant containing effective chlorine.

\section{Specialized practice recommendation:}

\section{Conservative dentistry and endodontic practice recommendation:}

1. Telemedicine and triage system for all patients: The individuals needing endodontic care should be reached via phone for the COVID-19 assessment. In case, if the individual reports the signs and symptoms reported by the CDC guidelines, the patient should be referred to the Primary care physician for further evaluation.
Following the evaluation, 1) If the tests are positive, avoid any elective endodontic treatment. Consider Scheduling this individual after two weeks only if the strict quarantine protocols were followed and the sign and symptoms have been diminished. Monitor them closely via phone. 2) If the tests are negative, consider an endodontic visit.

2. Defining Elective, Urgent, and Emergent care: Based on telemedicine, decide what is elective, urgent, and emergent care.

a. Elective care- If the individual has no dental-related signs and symptoms

b. Urgent care- If the individual has acute pain but can be diminished with analgesics and antibiotics

c. Emergent care- If the individual has acute pain with swelling with no response to the pharmacological intervention.

3. PPE awareness for auxiliary and patients: All the dental auxiliaries should be aware of the use of PPE to be safe and keep the dental office environment safe.

4. Operative sterilization and maintenance- Standard sterilization protocols and airborne precautions together, with the proper use of PPE and hand hygiene practices, should be incorporated in the endo-practice. The operating area should be in negative-pressure treatment rooms/ airborne infection isolation rooms to avoid contamination. Consider maintaining the operating area dry by using the disinfectants approved by CDC.

5. Preoperative mouth rinse- All the individuals undergoing endodontic treatment should consider rinsing the mouth with either hydrogen peroxide $(0.5 \%-1 \%)$ or povidone-iodine $(0.2 \%)$.

6. Instrument management- Consider using as many as possible disposable instruments for everyone. Consider extra oral radiographs to avoid gagging and coughing. In an urgent and complex case, if an intra-oral radiograph is required, consider having double barriers on the sensors. As a standard practice, use a rubber dam for individuals to avoid any splatters. Avoid using high-speed hand pieces to avoid splatter.

7. Follow up through telemedicine- Once the treatment is rendered, consider a close follow up through telemedicine and follow the same protocol if the individual requires another visit. 


\section{Oral medicine and Periodontology practice recommendation}

As the negative impact of COVID-19 infection on oral healthseems to be multidirectional, patients should be encouraged tomaintain safely their access to health systems, particularly in case of urgency/ emergency dental problems, and a clear concept for prioritizing procedures in oral medicine $(\mathrm{OM})$ and Periodontology (PD) with a careful triaging becomespivotal. It is important to note that postponed and delayed OM and PD care, deferring non-urgent cases (hypo salivation, oral lichen planus, oral candidiasis, and recurrent aphthous stomatitis RAS) may lead serious consequences for oral and systemic health, and could have a significant impact on the patients' well-being and quality of life $\mathrm{e}^{14,15}$. Deferred diagnosis of a malignant condition, such as oral cancer when suspected or even dysplasia in oral leucoplakia cases vastly implies the clinical outcome, influencing treatment and prognosis, subsequently ${ }^{16}$. Furthermore, chronic autoimmune disorders with oral manifestations, such as mucous membrane pemphigoid or pemphigus vulgaris, can be exacerbated as a result of delayed OM follow-ups, dental care and self-management or even interruption of long-term therapy ${ }^{17,18}$. In addition, the psychological stress-related issues during this COVID-19 situation cannot be ignored as this would potentially trigger the manifestation of periodontal diseases. Therefore, consistent and timely management of these $\mathrm{OM}$ and PD cases are crucial.

As proposed by The Royal College of Surgeons of England (RCSE), in this challenging COVID-19 scenario, it is recommended that virtual assistance to the patients and to the health care professional, particularly to the dentists, could help to prioritize the higher risk cases, avoiding face-to-face contact (RCSE 2020). In this way, only patients with highly suspicious malignant lesions would be referred to the oral medicine team in order to undergo clinical examination and appropriate procedures, such as an incisional biopsy. The oral medicine specialists should nowadays develop advanced skills online OM tele (video) consultations (TC) and remote triaging by setting up a self-care advice and reassurance. Telemedicine/tele dentistry (TM/TD) has been underrepresented and underestimated in normal circumstances, so far $^{19}$. Along these lines, the RCSE also suggested that prescribing service/consultant should manage by telephone consultation the patient's chronic therapies based on systemic corticosteroids or immunosuppressive (dosage adjustment, untoward events). New TM technology (Zoom, Teams, Skype videoconferencing software) may effectively support OM and PD specialists ${ }^{20}$, since the patient can submit photo or video of recorded oral mucosal problems, to be entered in the electronic health record, along with clinical notes gathered during TC. Because the main diagnostic value in $\mathrm{OM}$ and PD are related to visual assessment, these images and videos sent by patient can constitute a crucial element of provisional diagnosis, to give subsequent advice or urgent referral ${ }^{21}$. Even without of tactile assessment, TM can allow continuity of care, initial triaging, and prioritizing patients' needs. The RCSE recently recommended the involvement of two experienced OM clinicians in decision-making process, as an interim measure and result of ceases routine treatment planning (RCSE, 2020). This clinical protocol may prevent the occurrence of diagnostic errors and safeguard double verification of optimal standard of care during COVID-19 crisis.

However, in emergency cases, if the patients could not be diagnosed virtually and required physical oral examination, it should be done with utmost care considering the safety of both patient and dental professional. Preoperative antimicrobial mouth rinse could reduce the number of microbes in the oral cavity $^{22,23}$. Procedures that are likely toinduce coughing should be avoided (if possible) or performed cautiously ${ }^{24}$. Aerosol-generating procedures, such as the use of a 3-way syringe, should be minimized as much as possible. Intraoral $\mathrm{x}$-ray examination is the most common radiographic technique in dental imaging; however, it can stimulate saliva secretion and coughing ${ }^{25}$. Therefore, extra oral dental radiographies, such as panoramic radiography and cone beam $\mathrm{CT}$, are appropriate alternatives during the outbreak of COVID-19.

It is important to keep in mind that once the oral diagnosis is done and the tissue specimen collected and sent to the oral pathology laboratory, there will be a risk of diagnostic delay because it is expected that many are working partially. Therefore, diagnosis, treatment and surgical interventions should be well planned.

\section{Prosthodontic practice recommendation:}

Since dentists have to liaise with dental technicians for the preparation of dental prosthesis, it is important to ensure the maintenance of aseptic condition in dental laboratories. In this regard, effective communication and coordination between the dental clinic and commercial dental laboratory is indispensable. While taking impression for partial denture or complete denture, it is recommended to use disposable plastic or one time use impression tray with caution. However, if this is not possible thenautoclavableand reusable impression tray should be used. After every use the impression trays should be autoclaved at 121 $\operatorname{deg} \mathrm{C}$ and $100 \mathrm{kPa}$ pressure for 20 minutes prior next 
use. The paper prescriptions should be kept separate from wet impressions. The Impressions, prostheses and appliances must be cleaned first and then disinfected before transporting to the dental lab. Reciprocally, the completed devices, prosthesis and appliances when delivered to the patient from the dental lab, must be disinfected and free from contamination.It is very important to keep in mind that these transportations of dental prosthesis to and from the dental lab and the dental clinic are a potential source for disease transmission. Therefore, we have to remain very cautious about this.

Orthodontic practice recommendation:

Orthodontists, like other dental specialists may be at risk of acquiring COVID-19 through multiple transmission routes, e.g., droplets from sneezing and coughing or from orthodontic procedure, aerosols created orthodontic procedures, indirect contact where viral droplets fall on surface that the orthodontist later contacts, treating patients who may have experienced indirect contact transmission from replacing appliances, aligners and rubber bands, and being in contact with multiple such persons, including those who accompany the treatment receiver. ${ }^{26}$

COVID-19 pandemic demands the concept that all elective and routine orthodontic treatment should be suspended and only emergency treatment can be provided which includes impingement of orthodontic appliance into the gingiva or oral mucosa causing severe pain and or infection, circumstances related to dental trauma, or conditions where a lack of management would be harmful. (American Association of Orthodontist. COVID-19 Resources for Orthodontists 2020)

Orthodontists should try first to manage the emergency over the phone, preferably with written instructions. If possible, patient can be guided on how to manage minor emergencies at home providing them links to such information with audiovisual aids. ${ }^{26}$. The links may be available on orthodontic association websites (e.g., https://www1. aaoinfo.org/). Sometimes, over-the-counter medications may be prescribed. In case where such tele medicine and instructions cannot solve the emergency, procedures can be performed in clinic maintaining basic recommendations provided for dental practice.

Oral and maxillofacial surgery practice recommendation:

Oral and maxillofacial surgery during the COVID-19 pandemic can be categorized into
Emergency cases demand operation even in pandemic situation with taking all measures:

Severe bleeding due to maxillofacial injury or vascular lesion

Deep neck soft tissue infection withcompromised airway or potential to respiratory obstruction

Urgent cases need to be operated even in pandemic situation:

Expected airway obstruction due to neck infections Tracheostomy

Oral malignancy - wide excision, neck dissection and reconstruction

Orbital decompression where evidence of reduced visual acuity

Severe maxillofacial injury with lacerated soft tissue and facial bone fracture

Intermediate priority cases (elective surgery) can be deferred in pandemic situation:

Fracture facial bones where close reduction and fixation is adequate for treatment

Benign tumors of jaws and soft tissues

Larger cystic lesions

TMJ pathologies with pain

Low priority cases (elective surgery) can be deferred in pandemic situation:

TMJ pathologies without pain

Surgery for congenital cleft lip and palate

Secondary reconstructive procedures (free/pedicle flaps)

Procedures such as removal of teeth and related complications such as bleeding from extraction sockets, treatment of localized abscesses, repair of oro-antral fistulae, treatmentof pulpitis, etc. should be handled by general dentistry

Basic principles of oral and maxillofacial patient management:

1. Patients for surgery should be selected according to the nature of cases e.g. Emergency, urgent, intermediate and low priority (as mentioned above). Maxillofacial surgery represents an example of a specialty that has had to adapt to this outbreak, because of the subspecialties of oncology and traumatology

2. The basic principle is to consider all patients as Covid positive and precautions should be taken accordingly. 
3. RT PCR Covid screening should be done in all cases except in emergency.

4. Minimum staffs in the theatre, absence of surgical team during intubation, maintaining basic principles of Covid precautions by the anesthetist, quick surgical procedures by experts are recommended without compromising the quality of treatment delivery.

5. Meticulous surgery with avoidance of excessive bleeding and dirty environment, restricted use of electro cautery, following extra-oral approach in oral surgery are recommended.

6. Preventing aerosol generation should be prioritized by avoiding and preventing excessive cooling in drilling, using ultra sonic device, handpiece and saws (use of osteotome, chisel and mallet are encouraged)

7. Operation theatre protocols: A time interval of 15 min must pass after the patient has left the operating room before cleaning and disinfection can start. Also, waste management must follow well-defined rules ${ }^{27}$

8. Appropriate PPE should be worn during surgical procedures including wearing of N95/ FFP2/FFP3 respirator, full face shield or Powered Air-Purifying Respirator (PAPR). We realize that PAPRs may not be widely available, and other systems can be used such as the Stryker Flute system with a FFP3 respirator, or even N95 mask combined with goggles and hood would do the job as N95 and FFR (filtering face piece respirator) both have been accurately tested to filter at least $95 \%$ of 0.075 micrometer solid particle ${ }^{28}$ (WHO 2020b).

9. In the in-patient department, patient's attendant should be restricted and limited number of specialists and residents should be involved in patient care with follow up. Covid positive cases should be isolated and treated accordingly.

10. In outpatient department, all patients should be considered as Covid positive and protection should be taken. Admission should be restricted according to recommendation as stated.

\section{Special attention to Maxillofacial Injuries:}

1. Procedures should be performed by experienced surgeon with minimal number of assistants and OT staffs.
2. In general, closed reduction with MMF is recommended without compromising aesthesis and function to a greater extent.

3. Self-drilling is recommended over power drilling in monocortical screws or MMF screws. In case of drilling, water cooling and irrigation should be minimized or avoided.

4. Scalpel should get priority over monopolar cautery in mucosal or skin incisions

5. Bipolar cautery can be used for hemostasis on lowest power setting

6. Extra oral approach is better than intra oral approach by principle

\section{Special attention to oral cancer cases:}

Without treatment, some cancers could obstruct, others could metastasize cause earlydeath; how to manage patients with cancer while this pandemic goes on is tricky which could be based on "The risk-benefit calculation".

1. Individual patient decisions have to be made by multidisciplinary team members. The approaches through surgery, radiotherapy, chemotherapy in isolation or in combination should be based on expected outcome considering the disadvantages of surgery particularly in Covid situation.

2. If non-surgical therapy is equivalent to surgery and radiation, non-surgical therapy is recommended

3. Surgery is preferable especially for the cases with rapid progression, good prognostic early cancer, cases in which a worse outcome is expected if surgery is delayed, cancers with bleeding and impending airway and salvage surgery for recurrent or persistent disease with expected better outcome.

4. Primary closure or minimum reconstructive approaches are preferable over complicated procedures and free flaps to reduce the operative time.

5. Telemedicine services for reducing clinic visits, and switching to subcutaneous or oral Chemotherapies, rather than intravenous ones are recommended when possible.

\section{Conclusion:}

For protecting dental health care providers, other staffs and patients, triaging patients over the 
telephone or video systems, room ventilation, appropriate PPE, sanitization of protective apparel is recommended. Dentists and related specialists should keep up to date with and have regard to the latest advice from Government and the health service authority. The position may change daily and therefore it is important to check that advice periodically.

\section{Recommended Future Initiatives:}

At present, few patients are being attended and treated. When the Covid situation is over or pandemic is shifted to endemic, a large number of patients will rush to the hospitals and clinics. So the authorities and health care providers should be prepared to face the patient load and service needs in near future. Duration of patient service hours with remuneration of health care providers can be considered in upcoming non Covid situation. Adequate manpower, logistics, increase, re distribution and utilization of proper resources should be considered in further planning with definitely paying attention to prevent corruption in these processes. For all these, the individual and institutional authorities can allocate more budgets for need based facilities.

Acknowledgment: The authors appreciate Dr. Mohammad Tajul Islam, Adjunct Professor of Public Health, North South University, Bangladesh for support in writing this manuscript.

Conflict of interest: The authors declare that they have no conflict of interest.

Informed consent: Formal consent was not required for this purpose.

Ethical approval: This article does not contain any studies with human or animals performed by the authors.

\section{References:}

1. Zhu N, Zhang D, Wang W, Li X, Yang B, Song J, Zhao X, Huang B, Shi W, Lu R (2020) A novel coronavirus from patients with pneumonia in China, 2019. N Engl J Med 382:727-733, https://doi.org/10.1056/ NEJMoa2001017. PMid:31978945, PMCid:PMC7092803

2. Li Q, Guan X,WuP,Wang X, Zhou L, Tong Y, Ren R, Leung KS, Lau EH, Wong JY (2020) Early transmission dynamics in Wuhan, China, of novel coronavirusinfected pneumonia. N Engl J Med 382:1199-1207, https://doi.org/10.1056/NEJMoa2001316.PMid:3199585 7, PMCid:PMC7121484

3. Tait, R (2020). 'Czechs get to work making masks after government decree', The Guardian, Available at: https://www.theguardian.com/world/2020/mar/30/czech s-get-to-work-making-masks-after-government-decree-c oronavirus.

4. WHO, 2020. WHO Virtual press conference on COVID-19 [WWW Document]. World Heal. Organ. https://www.who.int/ docs/default- source/coronaviruse/transcripts/who-audioemergenciescoronavirus- press-conference-full-and-final11mar2020.pdf (accessed 3.29.20).

5. Warnakulasuriya S. 2020. Protecting dental manpower from COVID-19 infection. Oral Diseases. 2020;00:1-4. https://doi.org/10.1111/odi.13410.PMid:32401373,PMC id:PMC7272980

6. Chan JFW, Yuan S, Kok KH, et al. A familial cluster of pneumonia associated with the 2019 novel coronavirus indicating person-to-person transmission: a study of a family cluster. Lancet 2020;395:514-23. https://doi.org/ 10.1016/S0140-6736(20)30154-9

7. Guan WJ, Ni ZY, Hu Y, et al. Clinical characteristics of 2019 novel coronavirus infection in China. medRxiv 2020. doi: https://doi. org/10.1101/2020.02.06.20020974

8. Harrel SK, Molinari J. Aerosols and splatter in dentistry: a brief review of the literature and infection control implications. J Am Dent Assoc 2004;135:429-37, https://doi.org/10.14219/jada.archive.2004.0207. PMid:15127864, PMCid:PMC7093851

9. Raghunath N, Meenakshi S, Sreeshyla H, et al. Aerosols in Dental Practice- A Neglected Infectious Vector. Br Microbiol Res J 2016;14:1-8., https://doi.org/ 10.9734/ BMRJ/2016/24101

10. Kampf G, Todt D, Pfaender S, et al. Persistence of coronaviruses on inanimate surfaces and its inactivation with biocidal agents. J Hosp Infect 2020. [Epub ahead of print], https://doi.org/10.1016/j.jhin.2020.01.022. PMid:32035997, PMCid:PMC7132493

11. Eggers M, Eickmann M, Zorn J. Rapid and Effective Virucidal Activity of Povidone-Iodine Products Against Middle East Respiratory Syndrome Coronavirus (MERS-CoV) and Modified Vaccinia Virus Ankara (MVA). Infect Dis Ther 2015;4:491-501., https://doi.org/10.1007/s40121-015-0091-9 PMid:26416214, PMCid:PMC4675768

12. To KW, Tsang OY, Yip CY, Chan KH, Wu TC, Chan JC (2020). Consistent Detection of 2019 Novel Coronavirus in Saliva. Clinical Infectious Diseases. $2020 \mathrm{Jul}$ 28;71(15):841-843. https://doi.org/10.1093/cid/ciaa149. PMid:32047895, PMCid:PMC7108139

13. Jin LJ, Lamster IB, Greenspan JS, Pitts NB, Scully C, \& Warnakulasuriya $S$ (2015). Global burden of oral diseases: Emerging concepts, management and interplay with systemic health. Oral Diseases, 22(7), 609-619. https://doi.org/10.1111/odi.12428.PMid:26704694

14. Arduno PG, Conrotto D and Broccoletti R (2020). The outbreak of Novel Coronavirus disease (COVID-19) caused a worrying delay in the diagnosis of oral cancer in north-west Italy: the Turin Metropolitan Area experience. Oral Dis, 10.1111/odi.13362. 
15. Jones D, Neal RD, Duffy SRG, Scot, SE, Whitaker KL, Brain K (2020). Impact of the COVID-19 pandemic on the symptomatic diagnosis of cancer: the view from primary care. Lancet Oncol, S1470-2045(20)30242-4.

16. Meng L. ,Hua, F., Bian, Z. (2020). Coronavirus Disease 2019 (COVID-19): Emerging and FutureChallenges for Dental and Oral Medicine. J Dent Res, https://doi.org/ 10.1177/0022034520914246.PMid:32162995 PMCid:PMC7140973

17. Martins-Chaves R.R.; Gomes, C.C.; Gomez R.S. (2020). Immunocompromised patients and coronavirus disease 2019: a review and recommendations for dental health care. Brazilian Oral Research, 34, e048.Epub May 15, 2020.https://dx.doi.org/10.1590/1807-3107bor2020.vol.34.0048.

18. Kasperkiewicz M, Schmidt E, Fairley JA, Joly P, Payne A.S, Yal, ML et al. (2020). Expert recommendations for the management of autoimmune bullous diseases during the COVID-19 pandemic.J EurAcad Dermatology Venereol, 25. doi.org/10.1111/jdv.16525.

19. Brailo V., Firriolo, F.J., Tanaka, T.I., et al. (2015). World Workshop on Oral Medicine VI: Utilization of Oral Medicine-specific software for support of clinical care, research, and education:current status and strategy for broader implementation. Oral Surg Oral Med Oral Pathol OralRadiol, 120(2), 172-184. https://doi.org/10.1016/ j.oooo.2015.02.484.PMid:25843941

20. Villa, A. Sankar, V., Shiboski, C. (2020), Tele(oral) medicine: a new approach during the, https://doi.org/ 10.1111/odi.13364.PMid:32307831, PMCid:PMC7264524 COVID-19 crisis. Oral Dis. Accepted Author Manuscript.

21. Bradley, M., Black, P., Noble, S., Thompson, R., Lamey, P.J. (2010). Application of teledentistryin oral medicine in a community dental service, N. Ireland. Br Dent J, 209(8), 399-404, https://doi.org/10.1038/sj.bdj. 2010.928. PMid:20966999

22. Kohn WG, Collins AS, Cleveland JL, Harte JA, Eklund KJ, Malvitz DM;Centers for Disease Control and Prevention. 2003. Guidelines for infectioncontrol in dental health-care settings-2003. https://www.cdc.gov/ mmwr/preview/mmwrhtml/rr5217a1.htm.https://doi.org/ 10.14219/jada.archive.2004.0019.PMid:14959873

23. Marui VC, Souto MLS, Rovai ES, Romito GA, Chambrone L, Pannuti CM.2019. Efficacy of preprocedural mouth rinses in the reduction of microorganismsin aerosol: a systematic review. J Am Dent Assoc. 150(12): 1015-1026.https://doi.org/10.1016/j.adaj.2019.06.024.P Mid:31761015

24. World Health Organization. 2020a. Clinical management of severe acute respiratory infection when novel coronavirus (2019-nCoV) infection is suspected: interim guidance [accessed 2020 July 17].

25. Vandenberghe B, Jacobs R, Bosmans H. 2010. Modern dental imaging: areview of the current technology and clinical applications in dental practice.EurRadiol. 20(11):2637-2655. https://doi.org/10.1007/s00330-010-18361.PMid:20544352

26. Wang Y, Zhou CC, Shu R, Zou J. [Oral health management of children during the epidemic period of coronavirus disease 2019 (Chinese)] Sichuan Da XueXueBao Yi Xue Ban. 2020; 51(2): 151- 154. Google Scholar.

27. World Health Organization: Safe management of wastes from health-care activities: a summary. Geneva: World Health Organization, 2017.

28. World Health Organization, 2020b. Advice on the use of masks in the context of COVID-19: interim guidance. (5 June, 2020). [accessed 2020, July], https://doi.org/10.15557/ PiMR.2020.0005

\section{Other Resources:}

- AO CMF International Task Force Recommendations on Best Practices for Maxillofacial Procedures during COVID-19 Pandemic,20 March 2020.

- Guidelines for the staff of the Faculty of Dental Sciences, University of Peradeniya, Kandy, Srilanka during COVID-19 outbreak, 24 April 2020.

- NHS guidance: https://www.england.nhs.uk/ coronavirus/ wp-content/ uploads/sites/52/2020/03/ specialty- guideacute treatment- cancer-23- march-2020.pdf.

- University of Washington School of Dentistry COVID-19 Clinic Protocol, March, 2020

- American Association of Orthodontist.COVID-19 Resources for Orthodontists2020.Available at: https://wwwl.aaoinfo.org/ covid-19/. Accessed April 5, 2020

- Recommendations for Oral Medicine During COVID-19 pandemic. Royal College of Surgeons of England, 2020. Available online: https://www.rcseng.ac.uk/dental-faculties/ fds/coronavirus/Accessed: 27/05/2020. 